\title{
Statistical mechanics of animal movement: Animals's decision-making can result in superdiffusive spread
}

\author{
Paulo F. C. Tilles ${ }^{1}$, Sergei V. Petrovskiii ${ }^{2}$ \\ Department of Mathematics, University of Leicester \\ Leicester, LE1 7RH, UK
}

\begin{abstract}
Peculiarities of individual animal movement and dispersal have been a major focus of recent research as they are thought to hold the key to the understanding of many phenomena in spatial ecology. Superdiffusive spread and long-distance dispersal have been observed in different species but the underlying biological mechanisms often remains obscure. In particular, the effect of relevant animal behavior has been largely unaddressed. In this paper, we show that a superdiffusive spread can arise naturally as a result of animal behavioral response to small-scale environmental stochasticity. Surprisingly, the emerging fast spread does not require the standard assumption about the fat tail of the dispersal kernel.
\end{abstract}

Keywords: composite random walk; environmental stochasticity; fat tails; movement behavior; anomalous diffusion

\footnotetext{
${ }^{1}$ On leave from: Instituto de Fsica de Sao Carlos, Universidade de Sao Paulo, Sao Carlos, Brazil.

${ }^{2}$ Corresponding author. Email: sp237@le.ac.uk, phone: +44 116252 3916, fax: +44 1162523915.
} 


\section{Introduction}

Statistical mechanics of ecological processes has been identified as a promising direction of research that can create a unifying framework for many diverse applications [1]. Animal dispersal is a key factor that underlies a variety of phenomena in spatial ecology $[2,3]$ where application of concepts and tools of statistical mechanics has proved to be especially useful and insightful $[4,5,6]$. A major focus of research has been on understanding of how far individual animals can travel over a given time or, equivalently, how fast the mean squared displacement (MSD), $\delta^{2}(t)=<(\mathbf{r}(t)-\mathbf{r}(0))^{2}>$ (where $\mathbf{r}(t)$ is the position of the animal at time $t$ ), can grow with time [5,7]. Whilst $\delta^{2} \sim t$ corresponds to the standard diffusion and to the Brownian motion as the underlying stochastic process $[8,9,10]$, the case $\delta^{2} \sim t^{\gamma}$ with $\gamma>1$ is conventionally referred to as the "superdiffusion" and is linked to Levy flights and Levy walks $[5,11,12]$.

In field observations or laboratory experiments, for technical reasons the position of individual animal(s) is usually recorded not continuously but with a finite time-resolution or time-step, say $\Delta t[7,10,13]$. In the corresponding time-discrete framework, individual animal movement is described by the dispersal kernel $\rho(l)$ that gives the probability distribution of the distance $l$ traveled by an animal during the time $\Delta t$. In the case of $\Delta t$ being sufficiently small, the distance $l$ can be regarded as a step made along the movement path over the given time. The product $\rho(l) d l$ gives the probability that an individual released at $t=0$ will, after the time $\Delta t$, be found at a distance between $l$ and $l+d l$ from the point of its release. A 'thin-tailed' dispersal kernel with the rate of decay at large $l$ being exponential or faster corresponds to the Brownian motion; a 'fat-tailed' kernel with the power-law rate of decay at large distances, $\rho(l) \sim l^{-1-\mu}$ where $0<\mu<2$, is associated with the Levy flight $[5,7,12,14]$. In the intermediate case $\mu \geq 2$, the random walk slowly converges to the Brownian motion in the multi-step limit. Because of the lower rate of decay, a fingerprint of the Levy flight is a much higher frequency of the longdistance travel compared to the Brownian motion. Understanding of the fat-tailed/longdistance dispersal is important as it may significantly affect the rate of biological invasions $[15,16]$, the rate of pathogens spread [17], conditions of species persistence in fragmented landscapes [18, 19], etc. This is one of the reasons why patterns of individual animal movement have been a focus of considerable interest [20, 21, 22, 23, 24, 25].

Fat-tailed kernels correspond to animal spread at a much faster rate than that predicted by the Brownian motion; the fatter the kernel, the faster is the growth of the MSD with time. A question arises here as to whether quantification of the rate of spread in terms of the MSD can still be meaningful for the Levy flights because, in a strict mathematical sense, kernels with the power-law decay do not possess a finite variance for $\mu \leq 2$. There are, however, several ways to overcome this difficulty. For instance, one can introduce a 
finite characteristic speed of the travel, thus turning Levy flights to Levy walks $[12,26]$. A similar approach is based on exclusion of very large steps (hence effectively truncating the kernel at large distances) as the probability of their occurrence in any finite sample is extremely small [14]. Alternatively, one can consider a pseudo-MSD by calculating the moment of a lower (fractional) order [5]. Interestingly, all these approaches result in the same relation between the exponent $\gamma$ in the rate of spread and the exponent $\mu$ in the rate of decay in the dispersal kernel, namely, $\gamma=2 / \mu$ (where $0<\mu \leq 2$ ). Correspondingly, the case $\mu=1$ results in the ballistic-like spread with $\delta^{2} \sim t^{2}$ and the case $\mu=2 / 3$ results in a turbulent-like spread with $\delta^{2} \sim t^{3}$. Evaluating the value of $\gamma$ is therefore a universal way to quantify the rate of animal dispersal across a broad range of different movement patterns.

There is now significant evidence $[27,28,29,30,31]$ that animals of different taxa may employ the superdiffusive movement pattern; however, little has been done to understand the mechanisms behind the Levy walks. This evokes the classical problem of revealing the process resulting in a given pattern $[1,32]$. The existing theoretical framework is largely descriptive and is mostly focused on the properties of the step length distribution, e.g. arguing whether it is fat-tailed or not. The animal itself is often considered quite schematically, i.e. as a particle or even as a "robot" [33], simply assuming that, at certain moments of time, the next travel distance is somehow drawn from a given distribution.

Meanwhile, one important factor that distinguish animals from particles is behavior. Particles cannot make decisions but animals can [34, 35]. Behavior has indeed been identified as an essential factor affecting the movement pattern $[28,36]$ but its impact on animal movement and dispersal has only been considered on large spatial and temporal scales, e.g. as corresponds to animal movement over a large foraging area. The main objective of this paper is to show that animal behavior exhibited on a much smaller scale of a single step along the path is a crucial factor that can affect the movement pattern. Specifically, we are going to show that, in a noisy environment, animal's basic reaction to external clues can turn the movement into superdiffusion.

During their movement, animals receive cues or 'signals' from their environment. Such cues can be of visual, auditory or olfactory origin. Through these cues, animals become aware of the presence of predators, of the availability of food (e.g. by perceiving an odor), of the presence of a mating partner (e.g. by detecting female pheromones), etc. The cues therefore bring information that is vital for the animal's well-being. The animals respond to the cues by making decisions about their movement; in their conceptual paper, Nathan et al. [37] referred to this situation as "why move". Exact timing of the cues is difficult or perhaps even impossible to determine though, as it is affected by many different factors, some of them being either unknown or having a large uncertainty, or being stochastic. For instance, the smell is brought in with the air flow which is often 
turbulent. Correspondingly, we assume that the timing of the cues' perception can be regarded as random. Altogether, the cues can be regarded as a small-scale environmental stochasticity.

The standard approach to animal movement [5, 10, 21] consider animals making 'steps' along their movement path so that, at certain moments of time, the length of the next step is drawn from a given probability distribution. It apparently implies that the moving animal makes a decision with regard to where it wants to be after a time $\Delta t$, i.e. about its next position on the movement path. Correspondingly, the animal has to possess certain cognitive powers enabling it to plan its future actions or future state (such as its next location), at least over a certain relatively small period of time. However, here we argue that this is hardly feasible. While some mammals like apes or dogs may be capable of some elementary planning, the existence of similarly complex behaviors in lower animals (e.g. invertebrates) is highly unlikely. To the best of our knowledge there is no evidence that invertebrates can exhibit a reaction more complicated than an immediate response to a stimulus. On the other hand, the response to a stimulus through an involuntary reflex action resulting in a muscle contraction is a common physiological property of animals [38]. We therefore assume that the decision taken by the animal as a response to the environmental cues has an immediate effect. Specifically, we assume that the decision is implemented by changing the magnitude of the force that the animal exerts in order to maintain its movement.

In the next section, we translate the above assumptions into a model and undertake a comprehensive study of the properties of the corresponding movement pattern.

\section{Model and results}

The animal movement as a response to external signals or cues may be formally described as a sequence of bouts. A bout ends (and the next bout starts) when the animal receives a cue from the environment. We are considering the situation when the signals are distributed in time homogeneously (in the statistical sense) so that the mean number of cues received during a time interval depends only on its duration, say $t$, but not on its starting moment. With the further assumption that for a small $t$ the probability of receiving more than one cue is negligible, we have a situation that coincides precisely with the one resulting in the Poisson distribution. If we denote by $\omega$ the mean number of cues per unit time, then the probability that an animal receives at least one cue during the time $t$ is

$$
P_{t}(\omega)=1-e^{-\omega t} .
$$


Here parameter $\omega$ accounts for the properties of environmental noise but it is also related to the biology of the dispersing animals, because it depends on their perception threshold.

Equation (1) gives the probability that the movement will be interrupted during the time interval $(0, t)$, i.e. that the duration of the given bout will not exceed $t$. In order to obtain the probability density of bout duration, we need to differentiate Eq. (1) with respect to $t$, which results in the following exponential distribution:

$$
\psi(t)=\omega e^{-\omega t}
$$

Correspondingly, $\langle t>=1 / \omega$ is the characteristic time between the subsequent cues.

Upon receiving a cue, the animal changes its movement by exerting a force, i.e. by changing its acceleration. It is reasonable to expect that the animal tries to optimize its energy expense, and hence it is only accelerating for a certain time, say $\tau$ (which can be regarded as a typical time of the behavioral response), as is required to change its speed to a new value, and moves with an approximately constant speed after that until it receives another cue; see Fig. 1a. It is readily seen that, in terms of this framework, the usual description of the animal movement by the probability distribution of the movement velocities (e.g. see [39]) corresponds to the limiting case $\tau \ll 1 / \omega$, i.e. the animal adjusts its speed very fast compared to the average time between two subsequent cues. This case can be regarded as the movement in a low-noise 'passive' environment where the cues are rare.

(a)

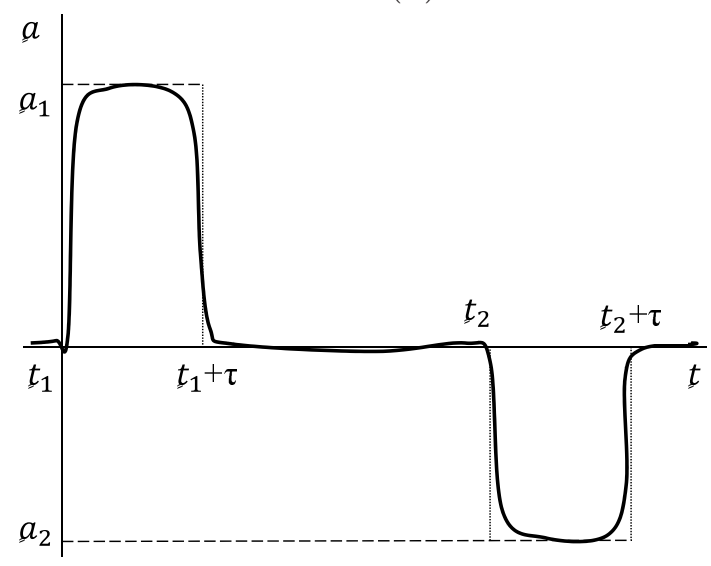

(b)

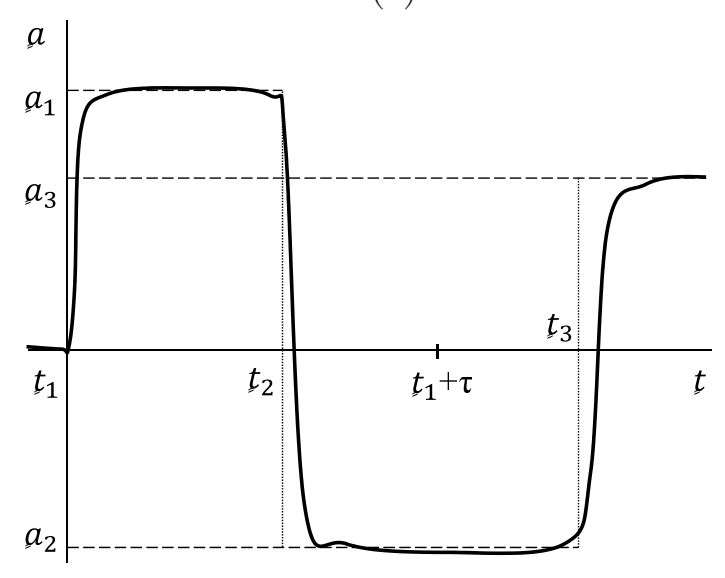

Figure 1: A sketch of the animal response to the environmental cues. The thick solid curve shows a hypothetical dependence of the acceleration $a$ on time, $t_{k}$ is the timing of the $k$ th cue, $\tau$ is the characteristic time of the behavioral response. (a) Acceleration stops when the behavior response is complete. (b) The case where $\tau \gg 1 / \omega$; acceleration changes to a new value when the next cue is received, i.e. before the behavioral response is complete. 
However, another biologically meaningful limiting case is the movement in a high-noise 'active' environment where the cues are relatively frequent, so that $\tau \gg 1 / \omega$. In this case, the next cue comes before the animal has enough time to adjust its speed; see Fig. 1 b. Correspondingly, the animal accelerates all the time between any two subsequent cues. The precise dependence of the acceleration on time is likely to be complicated; for the sake of simplicity, here we assume that it is approximately constant between any two subsequent cues (as shown by the values $a_{1}, a_{2}$ and $a_{3}$ in Fig. 1).

Therefore, under above assumptions, each bout is completely determined by its duration and the exercised acceleration through each bout. Note that these two variables are of different origin and hence are considered to be statistically independent. We will call the corresponding movement the composite random walk.

Since the environmental cues are considered to be stochastic, the force exerted by the animal is stochastic as well. The corresponding value $a$ of the acceleration is therefore described by a certain probability distribution $\phi(a)$. It seems reasonable to expect that, whatever is the cue, the animal tries to minimize its energy expense so that the most probable value is $a=0$. It is also reasonable to assume that the average energy expense is finite, so that $\phi(a)$ decays sufficiently fast at large $|a|$.

It is not possible to do the analysis in a general case of unspecified $\phi$. We therefore consider two particular cases of $\phi(a)$ satisfying the above requirements, namely, the Gaussian and Laplace probability distributions:

$$
\begin{array}{ll}
\text { (a) } \phi_{G}(a)=\frac{e^{-\frac{a^{2}}{2 \sigma^{2}}}}{\sqrt{2 \pi \sigma^{2}}}, & \text { (b) } \phi_{L}(a)=\frac{e^{-\frac{|a|}{\alpha}}}{2 \alpha} .
\end{array}
$$

In order to make these two cases comparable, we require them to have the same variance $<a^{2}>$ and choose the parameters $\alpha$ and $\sigma$ accordingly, so that $\sigma=\alpha \sqrt{2}$.

We begin with 1D case when $\delta^{2}(t)=<x^{2}(t)>($ assuming $x(0)=0$ without any loss of generality). The animal's position and the movement speed reached after the $k$ th bout of the duration $t_{k}$ are, respectively,

$$
x_{k}=x_{k-1}+v_{k-1} t_{k}+\frac{a_{k} t_{k}^{2}}{2}
$$

and

$$
v_{k}=v_{k-1}+a_{k} t_{k}
$$

where $k=1,2, \ldots$. Note that the increment $\Delta x_{k}=x_{k}-x_{k-1}$ of the animal's position is not independent of the previous one as it contains the speed reached in in the $(k-1)$ th bout. Therefore, animal's response to the environmental stochasticity by changing its movement acceleration results in a correlated random walk (CRW). We mention here that a stochastic process similar to (5) (but describing the turning speed) has been previously used to successfully model fish movement [40]. 
Once the probability densities for the bout duration and for the acceleration are defined, it is straightforward to generate the movement path; see Fig. 2. Contrary to the broken-line approximation commonly used in animal movement studies [5, 10, 33], the path obtained as a result of the composite random walk is smooth. We also note that the composite random walk considered here is time-continuous and hence is a valid framework for considering the individual movement on any time-scale $t$, small $(t \omega \ll 1)$ or large $(t \omega \gg 1)$.

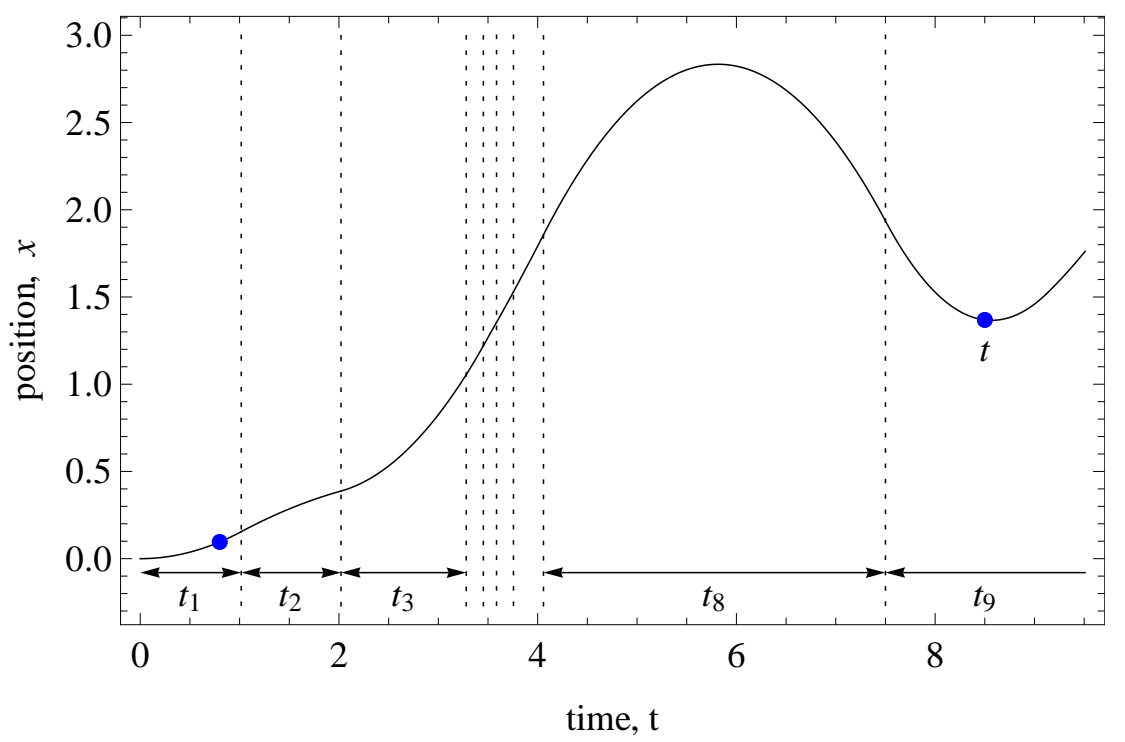

Figure 2: A sample path obtained for the bout duration distribution (2) with $\omega=1$ and the acceleration distribution (3a) with $\sigma=1 / 2$. The large black dots denote two different situations, i.e. when the observation time $t$ is small (within a single bout) and when it is large (contains several bouts).

For any given $t$, by averaging the corresponding animal position $x$ over many path realizations, one can calculate numerically the dispersal kernel $\rho(x, t)$ which gives the probability density that at time $t$ the animal will be located in the interval between $x$ and $x+d x$. Figure 3 shows the dispersal kernel obtained for $t=2$ for the two cases given by Eqs. (3a) and (3b). It appears that the asymptotical rate of decay of the emerging dispersal kernels follows closely that of the corresponding acceleration distribution.

Intuitively, the width of the dispersal kernel (e.g. as quantified by $<x^{2}>$ ) should grow with time, and this is indeed what is observed in simulation results obtained for other values of $t$ (not shown here for the sake of brevity). A crucial issue that determines the type of movement is the rate of growth. It appears possible to obtain the exact analytical expression for the MSD using methods of the statistical mechanics; see Appendix for details. For the composite random walk with the Gaussian distribution of acceleration, 


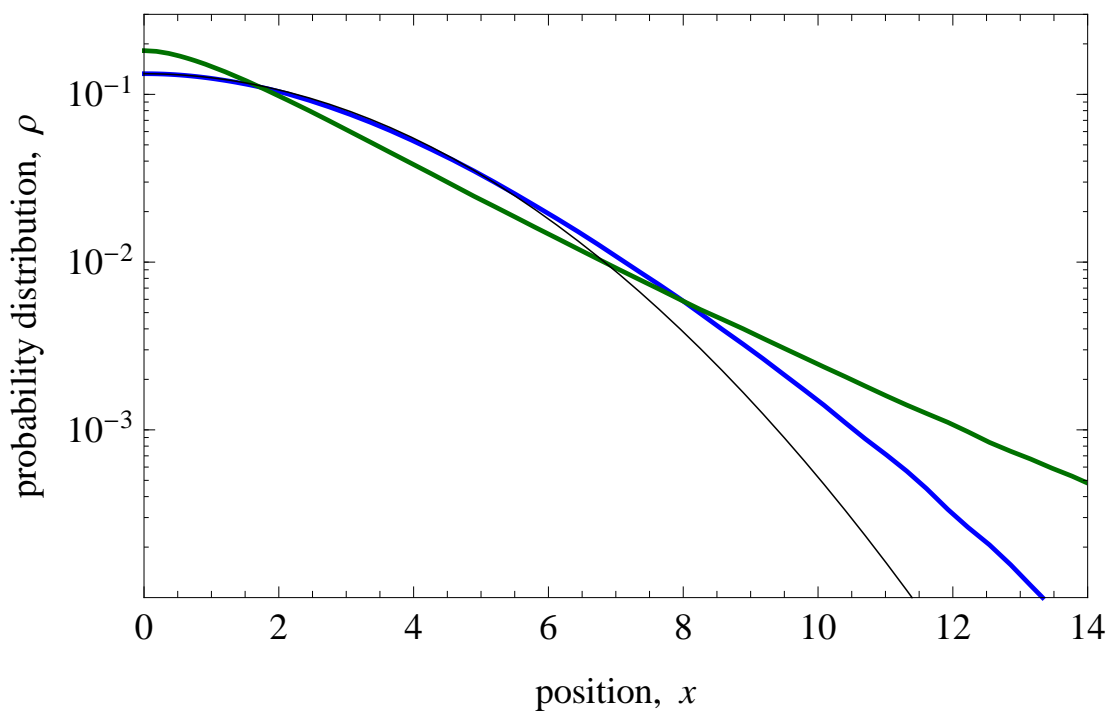

Figure 3: Probability density $\rho$ of the animal position (i.e. the dispersal kernel) calculated at $t=2$ from $10^{7}$ sample paths for $\omega=1$; in semilogarithmic coordinates. The blue curve shows the dispersal kernel arising from the Gaussian distribution of the acceleration (3a) with $\sigma=2$, the black curve is a baseline Gaussian distribution fitted to the same maximum value (at $x=0$ ) as the blue curve. The green curve corresponds to the the Laplace distribution with $\alpha=\sigma / \sqrt{2}=\sqrt{2}$.

see Eq. (3a), we obtain:

$$
\left\langle x^{2}(t)\right\rangle_{G}=\frac{\sigma^{2}}{3 \omega^{4}}\left[6-\omega^{2} t^{2}(3-2 \omega t)\right]-\frac{2 \sigma^{2}}{\omega^{4}} e^{-\omega t}(1+\omega t) .
$$

In particular, it is readily seen from (6) that $\left\langle x^{2}>\sim t^{4}\right.$ for small $t$ and it converges to a super-ballistic, turbulent-like spread with $<x^{2}>\sim t^{3}$ for large $t$.

In the above, our analysis was focused on the $1 \mathrm{D}$ case. However, the extension of the results onto a more realistic $2 \mathrm{D}$ case is straightforward. Indeed, since

$$
\boldsymbol{a}^{2}=a_{x}^{2}+a_{y}^{2}
$$

where $a_{x}$ and $a_{y}$ are the acceleration components, $\boldsymbol{a}=\left(a_{x}, a_{y}\right)$, for the $2 \mathrm{D}$ case we obtain:

$$
\phi(\boldsymbol{a})=\frac{e^{-\frac{a_{x}^{2}}{2 \sigma^{2}}}}{\sqrt{2 \pi \sigma^{2}}} \frac{e^{-\frac{a_{y}^{2}}{2 \sigma^{2}}}}{\sqrt{2 \pi \sigma^{2}}},
$$

which means that the displacement in the $x$ and $y$ direction goes independently, and hence $\left.<y^{2}\right\rangle$ is given by exactly the same expression as (6). For the MSD in the 2D case, we therefore obtain:

$$
\begin{aligned}
\delta^{2}(t)=\left\langle\mathbf{r}^{2}(t)\right\rangle_{G} & =\left\langle x^{2}(t)\right\rangle_{G}+\left\langle y^{2}(t)\right\rangle_{G} \\
& =\frac{4 \sigma^{2}}{\omega^{4}}\left[1-e^{-\omega t}(1+\omega t)-\frac{1}{2} \omega^{2} t^{2}+\frac{1}{3} \omega^{3} t^{3}\right]
\end{aligned}
$$


so that $\delta^{2} \sim t^{4}$ for small $t$ and $\delta^{2} \sim t^{3}$ for large $t$.

For the composite random walk resulting from the Laplace distribution (3b), the MSD appears to be described by the same expression as (6) subject to the relation between the distribution parameters $\sigma$ and $\alpha$ :

$$
\left\langle x^{2}(t)\right\rangle_{L}=\left.\left\langle x^{2}(t)\right\rangle_{G}\right|_{\sigma=\alpha \sqrt{2}}
$$

see Appendix for details. It indicates that the superdiffusive spread $<x^{2}>\sim t^{3}$ for large $t$ does not depend on the particular choice of the acceleration distribution $\phi(a)$ as long as it decays sufficiently fast at large $a$.

\section{Concluding remarks}

Existing theoretical approaches to individual animal movement consider animals in a very schematic, mechanistic way, i.e. as simple particles [23, 24] or robots [33], thus almost completely ignoring animal behavior. Here we argue that animal's behavioral response ('decision-making') to the small-scale stochasticity which is invariably present in every ecosystem as well as in laboratory can be a factor that determines the rate of their spread. We have considered a simple model where the animal reacts to the environmental cues or signals by exerting a force, hence changing the acceleration of their movement, and showed that the corresponding spread possesses superdiffusive properties.

Dispersal of animals going faster than that predicted by the standard diffusion model has been observed in species from different taxa and in different environments [7, 31] as well as in laboratory experiments [29]. The factors and processes behind this phenomenon are not always well understood though. The fast spread or "superdiffusion" is thought to be an inherent property of the long-distance fat-tailed dispersal, so that the power law in the probability density of the long-distance travel, i.e. $\rho(l) \sim l^{-1-\mu}$ where $0<\mu<2$, results in the MSD growing faster than linear, i.e. $\delta^{2} \sim t^{\gamma}$ where $\gamma=2 / \mu[5,26]$.

Evidence of a superdiffusive spread is now abundant, either inferred from the movement data directly (e.g. showing the faster than linear growth of the MSD with time) or indirectly, i.e. by relating the rate of spread to the rate of decay in the probability distribution of the dispersal distance. For instance, $\gamma=1.8$ was obtained for foraging albatrosses [7]. Dispersal kernel for Magellanic penguins shows the power-law decay rate with $\mu=0.7$ [31], apparently corresponding to $\gamma=2.86$ which is very close to the value $\gamma=3$ that we obtained in this paper. In the laboratory experiments on Tenebrio beetles, Reynolds at al. [29] obtained that the corresponding dispersal kernel has a very fat tail with $\mu=4 / 3$. Assuming that the relation between the dispersal kernel tail and the MSD is still valid, that would result in the super-turbulent spread with $\gamma=6$. This very large value is hardly possible for movement with a constant speed at each step. However, it 
may become possible if the beetles perform an accelerated movement; indeed, importance of the acceleration as a component of the beetle's curvilinear movement was mentioned in $[29]$.

Note that the relation between the scaling exponents $\gamma$ and $\mu$ is only valid under certain conditions that do not necessarily hold in animal dispersal. In particular, if the individual differences between the dispersing animals are taken into account, then the fat tail of the dispersal kernel (with $\mu<2$ ) does not necessarily result in a superdiffusive spread $[42,43]$. In this paper, we have shown that the inverse is not necessary either, i.e. the superdiffusive spread can occur with a thin-tailed dispersal kernel, in particular when the animal regulates its movement as a response to the small-scale stochasticity.

One of the main challenges in ecology is to explain the observed macroscopic pattern by linking it to the underlying processes that often go on a much smaller spatial and/or temporal scale. Correspondingly, "the challenge is to develop a statistical mechanics for such systems, connecting the microscopic and the macroscopic" [1]. In this paper, we have shown how the methods of statistical mechanics can be used to provide a possible explanation of animal's movement patterns observed on the macroscale, i.e. superdiffusive spread, by relating it to the effect of small-scale stochasticity and animal behavior. Specifically, we have shown that a decision-making by moving animals, considered here in a hypothetical way as the exertion of force to change their movement as a response to environmental clues, can result in a turbulent-like, super-ballistic movement.

We mention here that the fact that correlated steps can result in superdiffusive movement is not by itself new; one well known example is given by the fractional Brownian motion [44]. However, in this paper, our aim is not to construct another example of an autocorrelated motion, but rather to reveal the factors that may result in the autocorrelation. In particular, our aim is to show that animal behavioral response can result in the correlated random walk. Exerting muscle force as a response to external stimuli is one of the very basic physiological reactions [45]. Once a stimulus occurs and a force is exerted during the movement, by virtue of the Newton law the animal moves with acceleration as long as the force is being exerted.

Our model readily applies to the case where the animal receives the environmental cues sufficiently often, which means that the average bout duration is short compared to the characteristic time of the behavioral reaction. In its turn, a higher frequency of the cues may be characteristic for particular environment or weather types with a high level of the environmental noise. For instance, it seems plausible to expect that in the tropical jungle signals received from the environment are more frequent than in the arctic tundra, and the windy weather is likely to bring cues more frequently than the calm weather. It may also reflect the internal state of the animal. For instance, an animal that has been displaced into a new environment (e.g. as a result of capture-release) is likely to be 'on 
alert' and hence to have a lower perception threshold and to receive cues more frequently.

Note that, in this study, we assumed that the animal is in continuous movement. It apparently implies that the movement occurs on a relatively short time-scale (which, in particular, rules out unrealistically high values of speed that otherwise might be gained in the course of continuing acceleration). Indeed, there is considerable evidence that the distribution of periods of continuous movement is bounded by an exponential tail [13, 22]. If considered on a longer time-scale, periods of continuous movement would alternate with periods of rest. Once the rest times are included into consideration, intuitively, one can expect that the rate of spread should slow down. A rigorous analysis of this issue is technically challenging and will become a focus of future research.

\section{Acknowledgements}

We are thankful to Simon Levin (Princeton) for his encouraging comments on an earlier version of the manuscript. Helpful remarks from three anonymous reviewers are appreciated. Andrew Morozov, an Editorial Board Member, acted as the Editor-in-Chief handling the review processes for this manuscript. P.F.C.T. was supported by grant no. 2013/07476-0, Sao Paulo Research Foundation (FAPESP). 


\section{Appendix: calculation of the mean squared displace- ment}

In order to determine the properties of the probability distribution of the position of the dispersing animal, we first notice that it arises as a composition of the statistical properties from all possible sequences of bouts. The composed bout structure enables the dispersal kernel to be written as

$$
\rho(x, t)=\sum_{k=1}^{\infty} \rho_{k}(x, t),
$$

where $\rho_{k}(x, t)$ is the contribution of a sequence of $k$ bouts. Within this approach, one has to determine how both the time duration and the acceleration relate to the spatial position $x$ through their probability distributions.

To obtain the probability distribution for $x$ we must define not only the number of bouts that took place at the observation time $t$, but also the precise pdf for the acceleration that will be used. If we start with the Gaussian distribution (the reason will become evident at the end), then for a single bout period this procedure is quite simple: if we assume the animal to be at rest when the movement starts, then from the kinetics relation $x=a t^{2} / 2$ we obtain a conditional probability

$$
\rho_{1}\left(x, t \mid t_{1}\right)=\sqrt{\frac{2}{\pi \sigma^{2} t^{4}}} e^{-\frac{2 x^{2}}{\sigma^{2} t^{4}}},
$$

which gives the spatial pdf as a function of the actual time $t$ at which the observation is made, conditioned on the duration $t_{1}$ of the first bout. Since the observation is made at a time smaller than the total bout duration (the bout is still occurring when the observation is made), to write the whole contribution we must consider all possible bout periods lasting at least $t$, i.e.,

$$
\begin{aligned}
\rho_{1}(x, t) & =\int_{t}^{\infty} \rho_{1}\left(x, t \mid t_{1}\right) \psi\left(t_{1}\right) d t_{1} \\
& =e^{-\omega t} \sqrt{\frac{2}{\pi \sigma^{2} t^{4}}} e^{-\frac{2 x^{2}}{\sigma^{2} t^{4}}} .
\end{aligned}
$$

In order to compute the contributions from higher number of bouts we need a general method for determining how the sequences of i.i.d. accelerations relates to the position $x$ and time $t$ at which the observation is made.

One way of addressing this problem is to consider the values of the kinematics variables at the end of each bout. If we denote by $x_{k}$ the position, $v_{k}$ the velocity and $a_{k}$ the acceleration, all taken at the end of the $k^{\text {th }}$ bout, then from the standard movement 
equations

$$
\begin{aligned}
& x_{k}=x_{k-1}+v_{k-1} t_{k}+\frac{a_{k} t_{k}^{2}}{2}, \\
& v_{k}=v_{k-1}+a_{k} t_{k},
\end{aligned}
$$

we are able to write the final position as

$$
x_{k}=\sum_{n=1}^{k} t_{n}\left(\frac{t_{n}}{2}+\sum_{j=n+1}^{k} t_{j}\right) a_{n},
$$

where $t_{k}$ is measured from the start of the $k^{\text {th }}$ bout, so rather than the total time, it describes the duration of the respective bout. Equation (15) shows that the final position is obtained from a linear combination of $a_{n}$, and since each coefficient only rescales the variance of its respective acceleration distribution, we know that $x_{k}$ is also normally distributed. Now we just have to find a way to connect this equation to the observation time $t$, located inside the period of the last bout, and thus satisfying $\sum_{n=1}^{k-1} t_{n}<t<$ $\sum_{n=1}^{k} t_{n}$. The trick to obtain an expression that describes the behavior of the spatial position $x$ at this time $t$ is to consider the last bout period in equation (15) to end precisely at $t$, which means that this equation will serve our purposes if we set $t_{k}=t-\sum_{n=1}^{k-1} t_{n}$. As a result, we may write the conditional probability for $x$ as

$$
\rho_{k}\left(x, t \mid \mathbf{t}_{k-1}\right)=\frac{\exp \left[-\frac{x^{2}}{2 \Delta_{k}^{2}\left(t, \mathbf{t}_{k-1}\right)}\right]}{\sqrt{2 \pi \Delta_{k}^{2}\left(t, \mathbf{t}_{k-1}\right)}},
$$

where $\mathbf{t}_{k}=\left(t_{1}, \ldots, t_{k}\right)$, and the variance $\Delta_{k}^{2}$, obtained directly from (15) as a sum of normally distributed random variables, may be written as

$$
\begin{aligned}
& \Delta_{k}^{2}\left(t, \mathbf{t}_{k-1}\right)=\frac{\sigma^{2}}{4}\left[\sum_{n=1}^{k-1} t_{n}^{4}+\left(t-\sum_{n=1}^{k-1} t_{n}\right)^{4}\right. \\
& +4 \sum_{\substack{n, m \\
m>n}}^{k-1} t_{n}^{3} t_{m}+4 \sum_{n=1}^{k-1} t_{n}^{3}\left(t-\sum_{m=1}^{k-1} t_{m}\right) \\
& +4 \sum_{\substack{n, m \\
m>n}}^{k-1} t_{n}^{2} t_{m}^{2}+4 \sum_{n=1}^{k-1} t_{n}^{2}\left(t-\sum_{m=1}^{k-1} t_{m}\right)^{2} \\
& \left.+8 \sum_{\substack{n, m, j \\
j>m>n}}^{k-1} t_{n}^{2} t_{m} t_{j}+8 \sum_{\substack{n, m \\
m>n}}^{k-1} t_{n}^{2} t_{m}\left(t-\sum_{j=1}^{k-1} t_{j}\right)\right] .
\end{aligned}
$$

The last step to obtain the whole contribution of a $k$-bout sequence consists on summing all possible bout durations that compose $\rho_{k}(x, t)$. If we realize that periods up 
to $t_{k-1}$ are all constrained on the interval $(0, t)$, their contribution is addressed via the multidimensional integral

$$
\int \mathcal{D} t_{k-1} \equiv \int_{0}^{t} d t_{1} \int_{0}^{t-t_{1}} d t_{2} \ldots \int_{0}^{t-\sum_{n=1}^{k-2} t_{n}} d t_{k-1}
$$

For the last bout we just have to make sure that it has a minimal length compatible with $t$, because the fact that we make the observation while the animal is still moving makes it superiorly unbounded. Since the conditional probability (16) does not depend on the last bout period, we may compute this integral directly and write the probability density as

$$
\begin{aligned}
& \rho_{k}(x, t)=\int \mathcal{D} t_{k-1} \rho_{k}\left(x, t \mid \mathbf{t}_{k-1}\right) \int_{t-\sum_{n=1}^{k-1} t_{n}}^{\infty} d t_{k} \prod_{m=1}^{k} \psi\left(t_{m}\right) \\
& =\omega^{k-1} e^{-\omega t} \int \mathcal{D} t_{k-1} \rho_{k}\left(x, t \mid \mathbf{t}_{k-1}\right)
\end{aligned}
$$

which should be inserted back in equation (11) to give the dispersal probability.

Unfortunately the integrals in equation (19) are too complex to be solved analytically, but we may use the fact that the conditional probability (16) is sufficiently simple to make spatial integrations, as in

$$
\int_{-\infty}^{\infty} x^{2 n} \rho_{k}\left(x \mid t, \mathbf{t}_{k-1}\right) d x=\frac{2^{n} \Gamma\left(n+\frac{1}{2}\right) \Delta_{k}^{2 n}\left(t, \mathbf{t}_{k-1}\right)}{\sqrt{\pi}},
$$

with $n=1,2, \ldots$, which we may use to obtain the moments $\left\langle x^{2 n}\right\rangle$ of the full distribution $\rho(x, t)$ as long as we're able to compute the bout period integrals with the kernel $\Delta_{k}^{2 n}\left(t, \mathbf{t}_{k-1}\right)$. If we consider the second moment, then we may write

$$
\begin{aligned}
\left\langle x^{2}(t)\right\rangle & =\sum_{k=1}^{\infty} \int_{-\infty}^{\infty} x^{2} \rho_{k}(x, t) d x \\
& =\frac{\sigma^{2}}{4} e^{-\omega t}\left[t^{4}+\sum_{k=2}^{\infty} \omega^{k-1} \mathcal{F}_{k}(t)\right],
\end{aligned}
$$

where the first part is the contribution from $\rho_{1}(x, t)$, directly computed from equation (13), and the contribution from higher bout sequences is represented by a summation over the function

$$
\mathcal{F}_{k}(t)=\int \mathcal{D} t_{k-1} \Delta_{k}^{2}\left(t, \mathbf{t}_{k-1}\right) .
$$

As expressed in equation (17), the variance is composed by 8 terms. When we consider each of them separately and compute the integrals up to $t_{1}$, then we can observe that the result is indeed a well behaved function of $k$, which becomes very predictable after the 
analysis of just the first few terms. Hence, after identifying these functions and gathering them altogether, equation (22) may be written as a rather simple expression,

$$
\begin{aligned}
\mathcal{F}_{k}(t)= & \int_{0}^{t} d t_{1} \frac{k(k-1)\left(t-t_{1}\right)^{k-2}}{3(k+2) !} \mathcal{P}_{k}\left(t, t_{1}\right), \\
\mathcal{P}_{k}\left(t, t_{1}\right)= & 4(2 k+5) t^{3}\left(t-4 t_{1}\right)+12(k+3)(k+4) t^{2} t_{1}^{2} \\
& +\left(3 k^{2}+17 k+26\right) t_{1}^{3}\left(t_{1}-4 t\right),
\end{aligned}
$$

which may summed over the $k$ index and subsequently integrated over $t_{1}$, as in

$$
\begin{aligned}
& \sum_{k=2}^{\infty} \omega^{k-1} \mathcal{F}_{k}(t)=\int_{0}^{t} d t_{1}\left\{\frac { e ^ { - \omega ( t _ { 1 } - t ) } } { 3 \omega ^ { 3 } } \left[24-12 \omega^{2}\left(t_{1}-t\right)^{2}\right.\right. \\
& \left.\left.-8 \omega^{3}\left(t_{1}-t\right)^{3}+3 \omega^{4} t_{1}^{2}\left(t_{1}-2 t\right)^{2}\right]+\frac{8\left[\omega\left(t_{1}-t\right)-1\right]}{\omega^{3}}\right\} \\
& =\frac{4 e^{\omega t}}{3 \omega^{4}}\left[6-\omega^{2} t^{2}(3-2 \omega t)\right]-\frac{8}{\omega^{4}}(1+\omega t)-t^{4} .
\end{aligned}
$$

After inserting this result back into equation (21) we obtain the exact expression for the second moment of the dispersal kernel:

$$
\left\langle x^{2}(t)\right\rangle=\frac{\sigma^{2}}{3 \omega^{4}}\left[6-\omega^{2} t^{2}(3-2 \omega t)\right]-\frac{2 \sigma^{2}}{\omega^{4}} e^{-\omega t}(1+\omega t) .
$$

Once we have shown the procedure for obtaining analytical results for the Gaussian distributed case, we may turn our attention to extend this approach to describe the Laplace distributed one. This example presents a little drawback, since we cannot write a general expression for the conditional probability after $k$ bouts, as we did in equation (16). Nevertheless, we may start with the $k=1$ case and obtain the higher cases by repeatedly applying the rules for summing Laplace distributed random variables. When performing this tedious procedure, it is quite straightforward to observe that the contributions from each sequence of bouts to the mean squared displacement $\left\langle x^{2}\right\rangle$ are almost the same as the ones obtained from the Gaussian distributed case, with the only difference being a scaling constant which is equal to 2 . But this is precisely the factor we obtain when we compare the expressions for the second moment obtained from the Gaussian and Laplace distributions when the parameters are set to be equal $(\alpha=\sigma)$, so it should somehow be expected that this composed movement would indeed preserve this feature. Hence, we may write the mean square displacement obtained from the Laplace distribution with the identity

$$
\left\langle x^{2}(\alpha)\right\rangle_{L}=\left\langle x^{2}(\sigma=\sqrt{2} \alpha)\right\rangle_{G},
$$

showing that equation (25) describes both cases as long as the relation $\sigma=\sqrt{2} \alpha$ between their defining parameters is satisfied. 


\section{References}

[1] Levin, S.A. Towards the marriage of theory and data. Interface Focus 2, 1-3 (2012).

[2] Bullock, J.M., Kenward, R.E. \& Hails, R.S. (eds.) Dispersal ecology (Blackwell, Oxford, 2002).

[3] Clobert, J.., Danchin, E., Dhondt, A.A. \& Nichols, J.D. (eds.) Dispersal (Oxford University Press, Oxford, 2001).

[4] Bartumeus, F., Fernandez, P., da Luz, M.G.E., Catalan, J., Sole, R.V. \& Levin, S.A. Superdiffusion and encounter rates in diluted, low dimensional worlds. Eur. Phys. J. Spec. Top. 157, 157-166 (2008).

[5] Viswanathan, G.M., da Luz, M.G.E., Raposo, E.P. \& Stanley, H.E. The physics of foraging: an introduction to random searches and biological encounters (Cambridge University Press, Cambridge UK, 2011).

[6] Lewis, M., Maini, P. \& Petrovskii, S.V. Dispersal, individual movement, and spatial ecology: a mathematical perspective. Springer Lecture Notes in Mathematics Vol. 2071 (Springer, Berlin, 2013).

[7] Viswanathan, G.M., Afanasyev, V., Buldyrev, S.V., Murphy, E.J., Prince, P.A. \& Stanley, H.E. Lévy flight search patterns of wandering albatrosses, Nature 381, 413415 (1996).

[8] Chorin, A.J. \& Hald, O.H. Stochastic tools in mathematics and science (Springer, New York, 2006).

[9] Kareiva, P.M. Local movement in herbivorous insecta: applying a passive diffusion model to mark-recapture field experiments. Oecologia 57, 322-327 (1983).

[10] Turchin, P. Quantitative analysis of movement (Sinauer, Sunderland, 1998).

[11] Klafter, J. \& Sokolov, I.M. Anomalous diffusion spreads its wings. Phys. World 18, 29-32 (2005).

[12] Shlesinger, M.F., Zaslavsky, G.M. \& Klafter, J. Strange kinetics. Nature 363, 31-37 (1993).

[13] Mashanova, A., Oliver, T.H. \& Jansen, V.A.A. Evidence for intermittency and a truncated power law from highly resolved aphid movement data. J. R. Soc. Interface 7, 199-208 (2010). 
[14] Sornette, D. Critical phenomena in natural sciences (2nd edition) (Springer, Berlin, 2004).

[15] Kot, M., Lewis, M.A. \& van der Driessche, P. Dispersal data and the spread of invading organisms. Ecology 77, 2027-2042 (1996).

[16] Shigesada, N. \& Kawasaki, K. [Invasion and the range expansion of species: effects of long-distance dispersal] Dispersal Ecology. [Bullock, J.M., Kenward, R.E. \& Hails, R.S. (eds.)] [350-373] (Blackwell, Oxford, 2002).

[17] Brockmann, D., Hufnagel, L. \& Geisel, T. The scaling laws of human travel. Nature 439, 462-465 (2006).

[18] Baguette, M. Long distance dispersal and landscape occupancy in a metapopulation of the cranberry fritillary butterfly. Ecography 26, 153-160 (2003).

[19] Levin, S.A., Cohen, D. \& Hastings, A. Dispersal strategies in patchy environments. Theor.Popul. Biol. 26, 165-180 (1984).

[20] Benhamou, S. How many animals really do the Lévy walk? Ecology 88, 1962-1969 (2007).

[21] Codling, E.A., Plank, M.J. \& Benhamou, S. Random walk models in biology. J. R. Soc. Interface 5, 813-834 (2008).

[22] Edwards, A.M., Phillips, R.A., Watkins, N.W., Freeman, M.P., Murphy, E.J., Afanasyev, V. \& Buldyrev, S.V. Revisiting Lévy flight search patterns of wandering albatrosses, bumblebees and deer. Nature 449, 1044-1048 (2007).

[23] Viswanathan, G.M., Raposo, E.P. \& da Luz, M.G.E. Lévy flights and superdiffusion in the context of biological encounters and random searches. Phys. Life Rev. 5, 133150 (2008).

[24] Reynolds, A.M. Bridging the gulf between correlated random walks and Lévy walks: autocorrelation as a source of Lévy walk movement patterns. J. R. Soc. Interface 7, 1753-1758 (2010).

[25] Turchin, P. Fractal analyses of animal movement: a critique. Ecology 77, 2086-2090 (1996).

[26] Shlesinger, M.F., West, B.J. \& Klafter, J. Levy dynamics of enhanced diffusion: application to turbulence. Phys. Rev. Lett. 58, 1100-1103 (1987). 
[27] Ramos-Fernández, G., Mateos, J.L., Miramontes, O., Cocho, G., Larralde, H. \& Ayala-Orozco, B. Lévy walk patterns in the foraging movements of spider monkeys (Ateles geoffroyi). Behav. Ecol. Sociobiol. 55, 223-230 (2004).

[28] Reynolds, A.M. Olfactory search behaviour in the wandering albatross is predicted to give rise to Lévy flight movement patterns. Animal Behav. 83, 1225-1229 (2012).

[29] Reynolds, A.M., Lepretre, L. \& Bohan, D.A. Movement patterns of Tenebrio beetles demonstrate empirically that correlated-random-walks have similitude with a Lévy walk. Sci. Rep. 3, 3158; DOI:10.1038/srep03158 (2013).

[30] Reynolds, A.M., Smith, A.D., Menzel, R., Greggers, U., Reynolds, D.R. \& Riley, J.R. Displaced honey bees perform optimal scale-free search flights. Ecology 88, 1955-1961 (2007).

[31] Sims, D., et al. Scaling laws of marine predator search behavior. Nature 451, 1098-102 (2008).

[32] Levin, S.A. The problem of pattern and scale in ecology. Ecology 73, 1943-1967 (1992).

[33] Gautestad, A.O. Lévy meets Poisson: a statistical artifact may lead to erroneous recategorization of Lévy walk as Brownian motion. Am. Nat. 181, 440-450 (2013).

[34] Rankin, C.H. From gene to identified neuron to behaviour in Caenorhabditis elegans. Nat. Rev. Genetics 3, 622-630 (2002).

[35] Sinn, D.L., Perrin, N.A., Mather, J.A. \& Anderson, R.C. Early temperament traits in an octopus (Octopus bimaculoides). J. Compar. Psychology 115, 351-364 (2001).

[36] Owen-Smith, N., Fryxell, J.M. \& Merrill, E.H. Foraging theory upscaled: the behavioural ecology of hebivore movement. Phil. Trans. R. Soc. B 365, 2267-2278 (2010).

[37] Nathan, R., Getz, W.M., Revilla, E., Holyoak, M., Kadmon, R., Saltz, D. \& Smouse, P.E. A movement ecology paradigm for unifying organismal movement research. PNAS 105, 19052-19059 (2008).

[38] Purves, D., Augustine, G.J., Fitzpatrick, D., Hall, W.C., LaManta, L.S., McNamara, J.O. \& Williamms, S.M. Neuroscience. Third Edition (Sinauer, Massachusetts, 2004).

[39] Othmer H.G., Dunbar, S.R. \& Alt, W. Models of dispersal in biological systems. J. Math. Biol. 26, 263-298 (1988). 
[40] Gautrais, J., Jost, C., Soria, M., Campo, A., Motsch, S., Fournier, R., Blanco, S. \& Theraulaz, G. Analyzing fish movement as a persistent turning walker. J. Math. Biol. 58, 429-445 (2009).

[41] Kareiva, P.M. \& Shigesada, N. Analyzing insect movement as a correlated random walk. Oecologia 56, 234-238 (1983).

[42] Petrovskii, S.V. \& Morozov, A.Y. Dispersal in a statistically structured population: fat tails revisited. Am. Nat. 173, 278-289 (2009).

[43] Petrovskii, S.V., Mashanova, A. \& Jansen, V.A.A. Variation in individual walking behavior creates the impression of a Lévy flight. PNAS 108, 8704-8707 (2011).

[44] Mandelbrot, B. \& van Ness, J.W. Fractional Brownian motions, fractional noises and applications. SIAM Review 10(4), 422-437 (1968).

[45] Grier, J.W. \& Burk, T. Biology of Animal Behaviour (Mosby Year Book, St. Louis, 1992). 